\title{
PERRON TYPE THEOREMS FOR SKEW-EVOLUTION SEMIFLOWS
}

\author{
Ciprian Preda, Sebastian Rămneanţu and Raluca Mureşan \\ West University of Timişoara, Romania
}

\begin{abstract}
In the present paper we obtain some results for the asymptotic behavior of a large class of evolution families. Our approach uses the admissibility method initiated by O. Perron in the 1930's but the test functions that we choose are different from those employed in the case of differential systems.
\end{abstract}

\section{INTRODUCTION}

In recent years there has been an increasing interest in the qualitative theory of evolution equations due to its applications in pure and applied sciences, as well as in the study of social and engineering sciences. Differential equations as part of this theory are being studied regarding the asymptotic behaviour of their solutions and exponential stability plays a central role.

The notion of exponential dichotomy and thus stability for linear differential equations was introduced by O. Perron in 1930 in [15]. In this classic paper Perron was the first to establish the connection between the solution of the equation

$$
\dot{x}(t)=A(t) x(t)
$$

and the associated inhomogeneous equation

$$
\dot{x}=A(t) x(t)+f(t)
$$

in finite dimensional spaces, where $f$ is a continuous and bounded function on $\mathbb{R}_{+}$. This idea was later developed by W. A. Coppel ([7]) and P. Hartman $([9])$ for differential systems in finite dimensional spaces and the paper

2010 Mathematics Subject Classification. 34D05, 34D09.

Key words and phrases. Linear skew-evolution semiflow, evolution cocycle, uniform exponential stability, nonuniform exponential stability, Perron method. 
was a starting point for many works in the field of asymptotic properties of dynamical systems.

The properties of exponential stability and dichotomy for linear differential equations has gained prominence since the appearance of two fundamental monographs due to J. L. Daleckij and M. G. Krein ([8]) and J. L. Massera and J. J. Schäffer ([13]).

Several generalizations of differential equations appeared during the years, like the concepts of semigroups, evolution families and skew-product semiflows. The last notion has the advantage that it represents an unified approach of all the other concepts.

In this paper we study asymptotic properties of skew-evolution semiflows on Banach spaces, which are generalizations of the notion of skew-product semiflow. The latter arises naturally when one considers the linearization along an invariant manifold of a dynamical system generated by a nonlinear differential equation (see [21], Chapter 4). The major difference between these two concepts consists in the fact that a skew-evolution semiflow depends on three variables, while the classic notion of skew-product semiflow depends only on two variables, therefore justifying a further study of asymptotic behaviors for skew-evolution semiflows in a more general setting, the nonuniform case (relative to the third variable). We mention the fact that the classical example of a linear skew-product semiflow is the one that is associated with the solutions of a nonautonomous differential equation $(A)$ mentioned above on a Banach space $X$.

The notion of skew evolution semiflows was introduced by M. Megan and C. Stoica in [14]. Other results concerning the asymptotic properties of skew evolution semiflows and in particular, characterizations of the connections between the exponential dichotomy, dichotomic spectrum and Morse decomposition were recently obtained by M. Rasmussen in [20].

The theory of skew-product semiflows is well represented in the literature by the papers due to C. Chicone and Y. Latushkin ([4]), S. N. Chow and H. Leiva ([5,6]), R. J. Sacker and G. R. Sell ([21]), Y. Latushkin and A. M. Stepin ([10]), Y. Latushkin and R. Schnaubelt ([11]).

Characterization for the asymptotic properties of skew-product semiflows were obtained by C. Preda, P. Preda, A. Petre ([16]) and P. Preda, A. Pogan, C. Preda in [17].

In this paper we present some results concerning nonuniform asymptotic properties of skew evolution semiflows, thus we would like to present several causes of nonuniform asymptotical behaviour of dynamical systems. A cause for the nonuniform behaviour of the solutions of the differential equation $(A)$ is the non-integral-boundedness of the coefficients $A(t), t \geq 0$ (i.e.sup $\int_{t}^{t+1}\|A(\tau)\| d \tau=\infty$ ) as illustrated by J. L. Daleckij and M. G. Krein ([8]). They proved in Theorem 5.1, page 127, that a differential system of type 
$(A)$, for which the pair $\left(\mathcal{C}_{00}\left(\mathbb{R}_{+}, X\right), \mathcal{C}\left(\mathbb{R}_{+}, X\right)\right)$ is admissible to the evolution family generated by $A$ and denoted by $\Phi$, has the property that there exist $N: \mathbb{R}_{+} \rightarrow \mathbb{R}_{+}^{*}$ and $\nu>0$ such that

$$
\left\|\Phi\left(t, t_{0}\right)\right\| \leq N\left(t_{0}\right) e^{-\nu\left(t-t_{0}\right)} \text { for all } t \geq t_{0} \geq 0 .
$$

Furthermore, an example from the same book (p. 131) shows that the nonuniform stability of $\Phi$ is due to the fact that $A(t), t \geq 0$, are not integralbounded. It is, therefore, essential for the coefficients of the differential system $(A)$ to be integral bounded in order to have uniform behaviour of the solutions. In [18], M. Reghiş indicated that another cause for the nonuniform asymptotic behaviour of the solutions of the differential system $(A)$ is the non-invariance to translations of the input-output spaces (according to the terminology in [18]). These sources of nonuniform asymptotic behaviour are also presented in the paper [12] by J. L. Massera and J. J. Schäffer in Theorems 5.1 and 5.2, pages 534 and 535 respectively.

We also mention the results of nonuniform exponential stability and dichotomy given and studied in the papers [1-3] by L. Barreira, D. Dragičević and C. Valls. In these papers the authors analyze evolution families (and semigroups) that are nonuniformly exponentially bounded, i.e. there exist $M: \mathbb{R}_{+} \rightarrow \mathbb{R}_{+}^{*}$ and $\omega \in \mathbb{R}$ such that

$$
\left\|\Phi\left(t, t_{0}\right)\right\| \leq M\left(t_{0}\right) e^{\omega\left(t-t_{0}\right)}, \text { for all } t \geq t_{0} \geq 0
$$

and obtain results of nonuniform exponental stability and dichotomy in terms of admissibility of some pairs of function spaces.

In the present paper we obtain some results of uniform and nonuniform behaviour for the Perron problem for the stability of evolution families, in general as in [8]. We therefore obtain a result for skew evolution semiflows in general with a technique different from that of the evolution families generated by differential equations. Also, we prove that in this case we can consider as an input-output space a subclass of the space $\mathcal{C}$, the space denoted by $\mathcal{C}_{00}=\left\{f \in \mathcal{C}: f(0)=0\right.$ and $\left.\lim _{t \longrightarrow \infty} f(t)=0\right\}$ obtaining the same type of behaviour as in the case of the evolution families generated by differential equations. This class is the most general and the stability result obtained unifies the results of such type that arose from the Perron problem for the stability of the dynamical systems described by evolution families.

\section{Preliminaries And notations}

Let $(X,\|\cdot\|)$ be a Banach space and $(\Theta, d)$ a metric space. We denote by $(\mathcal{B}(X),\|\cdot\|)$ the Banach space of all linear and bounded operators from $X$ into itself and we consider $\Delta=\left\{\left(t, t_{0}\right) \in \mathbb{R}_{+}^{2}: t \geq t_{0} \geq 0\right\}$.

Definition 2.1. The map $\sigma: \Theta \times \Delta \rightarrow \Theta$ is called evolution semiflow on $\Theta$ if it satisfies the following conditions: 
(i) $\sigma(\theta, t, t)=\theta$, for all $\theta \in \Theta$ and $t \in \mathbb{R}_{+}$;

(ii) $\sigma\left(\sigma\left(\theta, s, t_{0}\right), t, s\right)=\sigma\left(\theta, t, t_{0}\right)$, for all $(t, s),\left(s, t_{0}\right) \in \Delta$ and $\theta \in \Theta$;

(iii) $\left(\theta, t, t_{0}\right) \mapsto \sigma\left(\theta, t, t_{0}\right)$ is continuous on $\Theta \times \Delta$.

Definition 2.2. Let $\sigma$ be a semiflow. A strongly continuous evolution cocycle over an evolution semiflow $\sigma$ is a function $\Phi: \Theta \times \Delta \rightarrow \mathcal{B}(X)$ that satisfies the following conditions:

(i) $\Phi(\theta, t, t)=I$, for all $t \in \mathbb{R}_{+}$and $\theta \in \Theta$;

(ii) $\Phi\left(\sigma\left(\theta, s, t_{0}\right), t, s\right) \Phi\left(\theta, s, t_{0}\right)=\Phi\left(\theta, t, t_{0}\right)$, for all $(t, s),\left(s, t_{0}\right) \in \Delta$ and $\theta \in \Theta$

(iii) $\left(\theta, t, t_{0}\right) \mapsto \Phi\left(\theta, t, t_{0}\right) x$ is continuous on $\Theta \times \Delta$ for all $x \in X$.

If in addition the evolution cocycle $\Phi$ also satisfies the following condition

(iv) there are $M>0$ and $\omega \in \mathbb{R}$ such that

$$
\left\|\Phi\left(\theta, t, t_{0}\right)\right\| \leq M e^{\omega\left(t-t_{0}\right)}, \text { for all }\left(t, t_{0}\right) \in \Delta \text { and } \theta \in \Theta
$$

then it is uniformly exponentially bounded.

DeFINITION 2.3. The linear skew-evolution semiflow associated with the above cocycle is the dynamical system $\pi=(\Phi, \sigma)$ on $\varepsilon=(X, \Theta)$ defined by $\pi: X \times \Theta \times \Delta \rightarrow X \times \Theta$,

$$
\pi\left(x, \theta, t, t_{0}\right)=\left(\Phi\left(\theta, t, t_{0}\right) x, \sigma\left(\theta, t, t_{0}\right)\right) .
$$

EXAMPLE 2.4. Let us consider a skew-evolution semiflow $\pi=(\Phi, \sigma)$ and a parameter $\lambda \in \mathbb{R}$. We define the mapping $\Phi_{\lambda}: \Delta \times X \longrightarrow \mathcal{B}(Y)$, where $Y$ is a Banach space and $\mathcal{B}(Y)$ is the space of all bounded linear operator from $Y$ into itself.

$$
\Phi_{\lambda}\left(\theta, t, t_{0}\right)=e^{\lambda\left(t-t_{0}\right)} \Phi\left(\theta, t, t_{0}\right) .
$$

One can remark that $\pi_{\lambda}=\left(\Phi_{\lambda}, \sigma\right)$ also satisfies the conditions of Definition 2.3 , begin called $\lambda$-shifted skew-evolution on $X \times \Theta$.

Let us consider on the Banach space $Y$ the Cauchy problem

$$
\left\{\begin{array}{l}
\dot{y}(t)=A y(t), \quad t>0, \\
y(0)=y_{0},
\end{array}\right.
$$

where $A$ is an operator which generates a $C_{0}$-semigroup $S=\{S(t)\}_{t \geq 0}$. Then $\Phi(\theta, t, s) y=S(t-s) y$, where $t \geq s \geq 0,(\theta, y) \in \Theta \times X$ defines an evolution cocycle. Moreover, the mapping defined by

$$
\Phi_{\lambda}: \Delta \times X \longrightarrow \mathcal{B}(Y), \Phi_{\lambda}(\theta, t, s) y=S(t-s) y,
$$

where $S_{\lambda}=\left\{S_{\lambda}(t)\right\}_{t \geq 0}$ is generated by the operator $A-\lambda I$, is also an evolution cocycle.

EXAMPLE 2.5. Let $f: \mathbb{R}_{+} \longrightarrow \mathbb{R}_{+}^{*}$ be a decreasing function with the property that there exists $\lim _{t \rightarrow \infty} f(t)=a>0$. We denote by $\mathcal{C}=\mathcal{C}\left(\mathbb{R}_{+}, \mathbb{R}_{+}\right)$ the set of all continuous function $x: \mathbb{R}_{+} \longrightarrow \mathbb{R}_{+}$endowed with the topology 
of uniform convergence on compact subsets of $\mathbb{R}_{+}$, metrizable by means of the distance

$$
d(x, y)=\sum_{n=1}^{\infty} \frac{1}{2^{n}} \frac{d_{n}(x, y)}{1+d_{n}(x, y)}
$$

where

$$
d_{n}(x, y)=\sup _{t \in[0, n]}|x(t)-y(t)|
$$

If $x \in \mathcal{C}$, then for all $t \in \mathbb{R}_{+}$, we denote $x_{t}(s)=x(t+s), x_{t} \in \mathcal{C}$. Let $X$ be the in $\mathcal{C}$ of the set $\left\{f_{t}, t \in \mathbb{R}_{+}\right\}$. It follows that $(X, d)$ is a metric space. The mapping $\sigma: \Delta \times X \longrightarrow X, \sigma(\theta, t, s)=x_{t-s}$ is an evolution semiflow on $X$. We consider $y=\left(y_{1}, y_{2}\right) \in Y$. If $u: \mathbb{R}_{+} \longrightarrow \mathbb{R}_{+}^{*}$, then the mapping $\Phi_{u}: \Delta \times X \longrightarrow \mathcal{B}(Y)$ defined by

$$
\Phi_{u}(\theta, t, s)=\left(\frac{u(s)}{u(t)} e^{-\int_{s}^{t} x(\tau-s) d \tau} y_{1}, \frac{u(t)}{u(s)} e^{\int_{s}^{t} x(\tau-s) d \tau} y_{2}\right)
$$

is an evolution cocycle over $\sigma$ and $\pi=\left(\Phi_{u}, \sigma\right)$ is a skew-evolution semiflow.

We consider the following function spaces:

$$
\begin{aligned}
& \mathcal{C}\left(\mathbb{R}_{+}, X\right)=\left\{f: \mathbb{R}_{+} \rightarrow X, f \text { continuous and bounded }\right\} \\
& \mathcal{C}_{00}\left(\mathbb{R}_{+}, X\right)=\left\{f \in \mathcal{C}\left(\mathbb{R}_{+}, X\right): \lim _{t \rightarrow \infty} f(t)=f(0)=0\right\}
\end{aligned}
$$

with the norm $\left\|\left|f\left\|\mid=\sup _{t \geq 0}\right\| f(t) \|\right.\right.$. Obviously they are Banach spaces.

Definition 2.6. The pair of function spaces $\left(\mathcal{C}_{00}\left(\mathbb{R}_{+}, X\right), \mathcal{C}\left(\mathbb{R}_{+}, X\right)\right)$ is admissible to the skew-evolution semiflow $\pi=(\Phi, \sigma)$ if for every $f \in$ $\mathcal{C}_{00}\left(\mathbb{R}_{+}, X\right)$, the map $t \mapsto x_{f}(\theta, t)$ belongs to the function space $\mathcal{C}\left(\mathbb{R}_{+}, X\right)$ for all $\theta \in \Theta$, where $x_{f}: \Theta \times \mathbb{R}_{+} \rightarrow X$ is defined by

$$
x_{f}(\theta, t)=\int_{0}^{t} \Phi(\sigma(\theta, \tau, 0), t, \tau) f(\tau) d \tau .
$$

\section{UNIFORM EXPONENTIAL STABILITY}

In this section, the cocycles considered are uniformly exponentially bounded. Thus the main result of this section gives a sufficient condition for a different type of uniform exponential stability of linear skew-evolution semiflows.

LEMMA 3.1. Let $\pi=(\Phi, \sigma)$ be a linear skew-evolution semiflow on $\varepsilon=$ $(X, \Theta)$. If the pair of function spaces $\left(\mathcal{C}_{00}\left(\mathbb{R}_{+}, X\right), \mathcal{C}\left(\mathbb{R}_{+}, X\right)\right)$ is admissible to $\pi$, then there exists $k(\theta)>0$ such that

$$
\left\|x_{f}(\theta, \cdot)\right\| \leq k(\theta)\left\||f \||, \text { for all } f \in \mathcal{C}_{00} .\right.
$$


Proof. We consider $\mathcal{U}_{\theta}: \mathcal{C}_{00}\left(\mathbb{R}_{+}, X\right) \rightarrow \mathcal{C}\left(\mathbb{R}_{+}, X\right), \mathcal{U}_{\theta} f=x_{f}$. Obviously $\mathcal{U}_{\theta}$ is a linear operator. Let $f_{n} \rightarrow f$ in $\mathcal{C}_{00}\left(\mathbb{R}_{+}, X\right)$ and $\mathcal{U}_{\theta} f_{n} \rightarrow g$ in $\mathcal{C}\left(\mathbb{R}_{+}, X\right)$. We show that $\mathcal{U}_{\theta} f=g$.

We have that

$$
\begin{aligned}
\left\|\mathcal{U}_{\theta} f_{n}(t)-\mathcal{U}_{\theta} f(t)\right\| & =\| \int_{0}^{t} \Phi(\sigma(\theta, \tau, 0), t, \tau)\left(f_{n}(\tau)-f(\tau) d \tau \|\right. \\
& \leq \int_{0}^{t} \| \Phi(\sigma(\theta, \tau, 0), t, \tau)\left(f_{n}(\tau)-f(\tau) \| d \tau\right. \\
& \leq \int_{0}^{t} M e^{\omega(t-\tau)}\left\|f_{n}(\tau)-f(\tau)\right\| d \tau \\
& \leq t M e^{\omega}\left\|f_{n}-f\right\| \underset{n \rightarrow \infty}{\longrightarrow} 0, \text { for all } t \geq 0 .
\end{aligned}
$$

This proves that $\mathcal{U}_{\theta} f_{n} \underset{n \rightarrow \infty}{\longrightarrow} \mathcal{U}_{\theta} f$. But $\mathcal{U}_{\theta} f_{n} \rightarrow g$ in $\mathcal{C}\left(\mathbb{R}_{+}, X\right)$, which shows that $\mathcal{U}_{\theta} f=g$ and this implies that $\mathcal{U}_{\theta}$ is a closed operator. By the Closed Graph Principle there exists $k(\theta)>0$ such that

$$
\left\|\left|x _ { f } ( \theta , \cdot ) \left\|\left|\leq k(\theta)\left\||f \|| \text {, for all } f \in \mathcal{C}_{00}\left(\mathbb{R}_{+}, X\right) .\right.\right.\right.\right.\right.
$$

Throughout the following we assume that $k(\theta)$ is a constant independent of $\theta$, i.e. $k(\theta)=k$.

The following is the main result of the first section.

TheOrem 3.2. Let $\pi=(\Phi, \sigma)$ be a linear skew-evolution semiflow on $\varepsilon=(X, \Theta)$. If the pair of function spaces $\left(\mathcal{C}_{00}\left(\mathbb{R}_{+}, X\right), \mathcal{C}\left(\mathbb{R}_{+}, X\right)\right)$ is admissible to $\pi$, then there exist $N, \nu>0$ such that

$$
\left\|\Phi\left(\sigma\left(\theta, t_{0}, 0\right), t, t_{0}\right) x\right\| \leq N e^{-\nu\left(t-t_{0}\right)}\|x\|
$$

for all $\left(t, t_{0}\right) \in \Delta, \theta \in \Theta$ and $x \in X$.

Proof. Let $n \in \mathbb{N}^{*}$ be a positive integer, $t_{0}>0, \delta>t_{0}$ and $x \in X \backslash\{0\}$ such that $\Phi\left(\sigma\left(\theta, t_{0}, 0\right), t, t_{0}\right) x \neq 0$, for all $\theta \in \Theta$ and $t \geq t_{0}$.

We consider the function $\chi_{n}: \mathbb{R}_{+} \rightarrow \mathbb{R}$,

$$
\chi_{n}(t)=\left\{\begin{array}{ll}
0, & t \in\left[0, t_{0}\right] \\
n\left(t-t_{0}\right), & t \in\left(t_{0}, t_{0}+\frac{1}{n}\right) \\
1, & t \in\left[t_{0}+\frac{1}{n}, \delta\right] \\
1+\delta-t, & t \in(\delta, \delta+1) \\
0, & t \geq \delta+1
\end{array} .\right.
$$

It is obvious that $\chi_{n} \in \mathcal{C}_{00}$ and that $\left\|\left|\chi_{n} \|\right|=1\right.$.

Now let $f_{n}: \mathbb{R}_{+} \rightarrow X, f_{n}(t)=\chi_{n}(t) \frac{\Phi\left(\sigma\left(\theta, t_{0}, 0\right), t, t_{0}\right) x}{\left\|\Phi\left(\sigma\left(\theta, t_{0}, 0\right), t, t_{0}\right) x\right\|}$. It is easy to see that $f_{n} \in \mathcal{C}_{00}$ and that $\left\|f_{n}\right\| \mid=1$. 
We have that

$$
\begin{aligned}
x_{f n}(\theta, t)=\int_{0}^{t} \Phi(\sigma(\theta, \tau, 0), t, \tau) f_{n}(\tau) d \tau \\
=\int_{0}^{t} \chi_{n}(\tau) \frac{d \tau}{\left\|\Phi\left(\sigma\left(\theta, t_{0}, 0\right), \tau, t_{0}\right) x\right\|} \Phi(\sigma(\theta, \tau, 0), t, \tau) \Phi\left(\sigma\left(\theta, t_{0}, 0\right), \tau, t_{0}\right) x \\
=\int_{t_{0}}^{t} \chi_{n}(\tau) \frac{d \tau}{\left\|\Phi\left(\sigma\left(\theta, t_{0}, 0\right), \tau, t_{0}\right) x\right\|} \Phi\left(\sigma\left(\theta, t_{0}, 0\right), t, t_{0}\right) x .
\end{aligned}
$$

By Theorem 3.1 it follows that

$$
\left\|x_{f_{n}}(\theta, t)\right\| \leq k, \text { for all } \theta \in \Theta, t \geq 0 .
$$

Thus

$$
\int_{t_{0}}^{t} \chi_{n}(\tau) \frac{d \tau}{\left\|\Phi\left(\sigma\left(\theta, t_{0}, 0\right), \tau, t_{0}\right) x\right\|}\left\|\Phi\left(\sigma\left(\theta, t_{0}, 0\right), t, t_{0}\right) x\right\| \leq k .
$$

We put $t=\delta$ and we get that

$$
\int_{t_{0}}^{\delta} \frac{d \tau}{\left\|\Phi\left(\sigma\left(\theta, t_{0}, 0\right), \tau, t_{0}\right) x\right\|} \leq \frac{k}{\left\|\Phi\left(\sigma\left(\theta, t_{0}, 0\right), \delta, t_{0}\right) x\right\|} \text {, for all } \theta \in \Theta, \delta \geq t_{0} .
$$

This is equivalent to

$$
\int_{t_{0}}^{t} \frac{d \tau}{\left\|\Phi\left(\sigma\left(\theta, t_{0}, 0\right), \tau, t_{0}\right) x\right\|} \leq \frac{k}{\left\|\Phi\left(\sigma\left(\theta, t_{0}, 0\right), t, t_{0}\right) x\right\|} \text {, for all } \theta \in \Theta, t \geq t_{0} .
$$

We denote $\varphi: \mathbb{R}_{+} \rightarrow \mathbb{R}$

$$
\varphi(t)=\int_{t_{0}}^{t} \frac{d \tau}{\left\|\Phi\left(\sigma\left(\theta, t_{0}, 0\right), \tau, t_{0}\right) x\right\|} .
$$

We get that $\varphi(t) \leq k \dot{\varphi}(t)$, which is equivalent to

$$
\frac{1}{k} \leq \frac{\dot{\varphi}(t)}{\varphi(t)}, \text { for all } \theta \in \Theta, t>0
$$

Let $t \geq t_{0}+1$. By integrating the last relation on $\left[t_{0}+1, t\right]$, we have that

$$
\varphi\left(t_{0}+1\right) e^{\frac{1}{k}\left(t-t_{0}-1\right)} \leq \varphi(t)
$$

But

$$
\varphi\left(t_{0}+1\right)=\int_{t_{0}}^{t_{0}+1} \frac{d \tau}{\left\|\Phi\left(\sigma\left(\theta, t_{0}, 0\right), \tau, t_{0}\right) x\right\|}
$$

and

$$
\left\|\Phi\left(\sigma\left(\theta, t_{0}, 0\right), \tau, t_{0}\right) x\right\| \leq M e^{\omega\left(\tau-t_{0}\right)}\|x\| \leq M e^{\omega}\|x\|,
$$

which shows that

$$
\frac{1}{M e^{\omega}\|x\|} \leq \int_{t_{0}}^{t_{0}+1} \frac{d \tau}{\left\|\Phi\left(\sigma\left(\theta, t_{0}, 0\right), \tau, t_{0}\right) x\right\|}
$$


By relation (3.1) it follows that

$$
\frac{1}{M e^{\omega}\|x\|} e^{\frac{1}{k}\left(t-t_{0}\right)} e^{-\frac{1}{k}} \leq \frac{k}{\left\|\Phi\left(\sigma\left(\theta, t_{0}, 0\right), t, t_{0}\right) x\right\|}
$$

which is equivalent to

$$
\left\|\Phi\left(\sigma\left(\theta, t_{0}, 0\right), t, t_{0}\right) x\right\| \leq k M e^{\omega} e^{\frac{1}{k}} e^{-\frac{1}{k}\left(t-t_{0}\right)}\|x\| .
$$

If $t \in\left[t_{0}, t_{0}+1\right)$, then

$$
\left\|\Phi\left(\sigma\left(\theta, t_{0}, 0\right), t, t_{0}\right) x\right\| \leq M e^{\omega} e^{\frac{1}{k}\left(t-t_{0}\right)} e^{-\frac{1}{k}\left(t-t_{0}\right)}\|x\| \leq M e^{\omega} e^{\frac{1}{k}} e^{-\frac{1}{k}\left(t-t_{0}\right)}\|x\| .
$$

We denote by $N=M e^{\omega} e^{\frac{1}{k}} \max \{1, k\}$ and $\nu=\frac{1}{k}$, thus the last relation can be rewritten as follows:

$$
\left\|\Phi\left(\sigma\left(\theta, t_{0}, 0\right), t, t_{0}\right) x\right\| \leq N e^{-\nu\left(t-t_{0}\right)}\|x\|, \text { for all } \theta \in \Theta, t \geq t_{0} .
$$

Next we assume that for $x \in X \backslash\{0\}$ there exists $t^{\prime}>t_{0}$ such that

$$
\Phi\left(\sigma\left(\theta, t_{0}, 0\right), t^{\prime}, t_{0}\right) x=0
$$

Therefore

$$
\Phi\left(\sigma\left(\theta, t_{0}, 0\right), t^{\prime}, 0\right) \Phi\left(\sigma\left(\theta, t_{0}, 0\right), t^{\prime}, t_{0}\right) x=0
$$

and by the evolution property of the cocycle $\Phi$ we have that

$$
\Phi\left(\sigma\left(\theta, t_{0}, 0\right), t, t_{0}\right) x=0, \text { for all } t>t^{\prime} .
$$

We denote

$$
\mu=\inf \left\{t>t_{0}: \Phi\left(\sigma\left(\theta, t_{0}, 0\right), t, t_{0}\right) x=0\right\}
$$

and so $\Phi\left(\sigma\left(\theta, t_{0}, 0\right), \mu, t_{0}\right) x=0$. It follows that

$$
\left\|\Phi\left(\sigma\left(\theta, t_{0}, 0\right), t, t_{0}\right) x\right\| \leq N e^{-\nu\left(t-t_{0}\right)}\|x\|, \text { for all } t \in\left[t_{0}, \mu\right) .
$$

In conclusion we obtain

$$
\left\|\Phi\left(\sigma\left(\theta, t_{0}, 0\right), t, t_{0}\right) x\right\| \leq N e^{-\nu\left(t-t_{0}\right)}\|x\|, \text { for all } \theta \in \Theta, t \geq t_{0}, x \in X .
$$

REMARK 3.3. In Theorem 3.2, if $k=k(\theta): \Theta \rightarrow \mathbb{R}_{+}$, then there exist $N: \Theta \rightarrow \mathbb{R}_{+}^{*}$ and $\nu: \Theta \rightarrow \mathbb{R}_{+}^{*}$, given by $N(\theta)=M e^{\omega} e^{\frac{1}{k(\theta)}} \max \{1, k(\theta)\}$ and $\nu(\theta)=\frac{1}{k(\theta)}$ such that

$$
\left\|\Phi\left(\sigma\left(\theta, t_{0}, 0\right), t, t_{0}\right) x\right\| \leq N(\theta) e^{-\nu(\theta)\left(t-t_{0}\right)}\|x\|, \text { for all } \theta \in \Theta, t \geq t_{0}, x \in X
$$




\section{NONUNIFORM EXPONENTIAL STABILITY}

In all that follows we assume that the cocycles considered do not satisfy property $(i v)$, i.e. they are not uniformly exponentially bounded.

REMARK 4.1. Let $\pi=(\Phi, \sigma)$ be a linear skew-evolution semiflow on $\varepsilon=(X, \Theta)$. Then there exists $M_{\theta, t}>0$ such that

$$
\|\Phi(\theta, t, \tau) x\| \leq M_{\theta, t}\|x\|, \text { for all } \tau \in[0, t], \theta \in \Theta, x \in X .
$$

Indeed, let $\theta \in \Theta, t \geq 0, \tau \in[0, t]$ and $x \in X$. Since $\Phi(\theta, t, \tau) x$ is continuous on $[0, t]$, there exists $M_{\theta, t, x}>0$ such that

$$
\|\Phi(\theta, t, \tau) x\| \leq M_{\theta, t, x}, \text { for all } \tau \in[0, t] .
$$

By the Uniform Boundedness Principle there exists $M_{\theta, t}>0$ such that

$$
\|\Phi(\theta, t, \tau) x\| \leq M_{\theta, t}, \text { for all } \tau \in[0, t], x \in X .
$$

LEMma 4.2. Let $\pi=(\Phi, \sigma)$ be a linear skew-evolution semiflow on $\varepsilon=$ $(X, \Theta)$. If the pair of function spaces $\left(\mathcal{C}_{00}\left(\mathbb{R}_{+}, X\right), \mathcal{C}\left(\mathbb{R}_{+}, X\right)\right)$ is admissible to $\pi$, then there exists $k(\theta)>0$ such that

$$
\left\|\left|x _ { f } ( \theta , \cdot ) \left\|\left|\leq k(\theta)\left\||f \||, \text { for all } f \in \mathcal{C}_{00}\left(\mathbb{R}_{+}, X\right), \theta \in \Theta\right.\right.\right.\right.\right.
$$

Proof. Following the steps of the proof of Theorem 3.1 and by Remark 4.1, we obtain that

$$
\left\|\mathcal{U}_{\theta} f_{n}(t)-\mathcal{U}_{\theta} f(t)\right\| \leq t M_{\theta, t}\left\|\mid f_{n}-f\right\| \| \underset{n \longrightarrow \infty}{\longrightarrow} 0
$$

for all $\theta \in \Theta$ and $t>0$. It follows that $\mathcal{U}_{\theta} f=g$ and by the Closed Graph Principle there exists $k(\theta)>0$ such that

$$
\left\|\left|x_{f}(\theta, \cdot)\||\leq k(\theta)\|\mid f\| \|,\right.\right.
$$

for all $f \in \mathcal{C}_{00}\left(\mathbb{R}_{+}, X\right)$ and all $\theta \in \Theta$.

THEOREM 4.3. Let $\pi=(\Phi, \sigma)$ be a linear skew-evolution semiflow on $\varepsilon=(X, \Theta)$. If the pair of function spaces $\left(\mathcal{C}_{00}\left(\mathbb{R}_{+}, X\right), \mathcal{C}\left(\mathbb{R}_{+}, X\right)\right)$ is admissible to $\pi$, then there exist $N: \Theta \rightarrow \mathbb{R}_{+}^{*}$ and $\nu: \Theta \rightarrow \mathbb{R}_{+}^{*}$ such that

$$
\left\|\Phi\left(\sigma\left(\theta, t_{0}, 0\right), t, t_{0}\right) x\right\| \leq N\left(\theta, t_{0}\right) e^{-\nu(\theta)\left(t-t_{0}\right)}\|x\|,
$$

for all $t \geq t_{0}, \theta \in \Theta$ and $x \in X$.

Proof. Let $\delta>t_{0}, x \in X \backslash\{0\}$ such that $\Phi\left(\sigma\left(\theta, t_{0}, 0\right), t, t_{0}\right) x \neq 0$. We consider $\chi: \mathbb{R}_{+} \rightarrow \mathbb{R}_{+}$given in the proof of Theorem 3.2 and $f_{n}: \mathbb{R}_{+} \rightarrow X$,

$$
f_{n}(t)=\chi_{n}(t) \frac{\Phi\left(\sigma\left(\theta, t_{0}, 0\right), t, t_{0}\right) x}{\left\|\Phi\left(\sigma\left(\theta, t_{0}, 0\right), t, t_{0}\right) x\right\|} .
$$

Obviously $f_{n} \in \mathcal{C}_{00}\left(\mathbb{R}_{+}, \mathbb{X}\right)$ and $\||f \||=1$. Proceeding as in the proof of Theorem 3.2, we obtain

$$
\varphi\left(t_{0}+1\right) e^{\frac{1}{K(\theta)}\left(t-t_{0}-1\right)} \leq \varphi(t), \text { for all } t \geq t_{0}+1 .
$$


But

and

$$
\varphi\left(t_{0}+1\right)=\int_{t_{0}}^{t_{0}+1} \frac{d \tau}{\left\|\Phi\left(\sigma\left(\theta, t_{0}, 0\right), \tau, t_{0}\right) x\right\|}
$$

$$
\begin{aligned}
\left\|\Phi\left(\sigma\left(\theta, t_{0}, 0\right), \tau, t_{0}\right) x\right\| & \leq \sup _{\tau \in\left[t_{0}, t_{0}+1\right]}\left\|\Phi\left(\sigma\left(\theta, t_{0}, 0\right), \tau, t_{0}\right) x\right\| \\
& \leq \sup _{\tau \in\left[t_{0}, t_{0}+1\right]}\left\|\Phi\left(\sigma\left(\theta, t_{0}, 0\right), \tau, t_{0}\right)\right\|\|x\|=M\left(\theta, t_{0}\right)\|x\|,
\end{aligned}
$$

where $M\left(\theta, t_{0}\right)=\sup _{\tau \in\left[t_{0}, t_{0}+1\right]}\left\|\Phi\left(\sigma\left(\theta, t_{0}, 0\right), \tau, t_{0}\right)\right\|$. By the relation (4.1) we have that

$$
\frac{1}{M\left(\theta, t_{0}\right)\|x\|} e^{\frac{1}{k(\theta)}\left(t-t_{0}\right)} e^{-\frac{1}{k(\theta)}} \leq \frac{k(\theta)}{\left\|\Phi\left(\sigma\left(\theta, t_{0}, 0\right), t, t_{0}\right) x\right\|} .
$$

It follows that

$$
\left\|\Phi\left(\sigma\left(\theta, t_{0}, 0\right), t, t_{0}\right) x\right\| \leq M\left(\theta, t_{0}\right) k(\theta) e^{\frac{1}{k(\theta)}} e^{-\frac{1}{k(\theta)}\left(t-t_{0}\right)}\|x\|,
$$

for all $t \geq t_{0}+1$ and $\theta \in \Theta$. If $t \in\left[t_{0}, t_{0}+1\right)$, then

$$
\begin{aligned}
\left\|\Phi\left(\sigma\left(\theta, t_{0}, 0\right), t, t_{0}\right) x\right\| & \leq M\left(\theta, t_{0}\right) e^{-\frac{1}{k(\theta)}\left(t-t_{0}\right)} e^{\frac{1}{k(\theta)}\left(t-t_{0}\right)}\|x\| \\
& \leq M\left(\theta, t_{0}\right) e^{\frac{-1}{k(\theta)}\left(t-t_{0}\right)} e^{\frac{1}{k(\theta)}}\|x\| .
\end{aligned}
$$

We denote $N\left(\theta, t_{0}\right)=e^{\frac{1}{k(\theta)}} M\left(\theta, t_{0}\right) \max \{1, k(\theta)\}$ and $\nu(\theta)=\frac{1}{k(\theta)}$. Therefore we have obtained that

$$
\left\|\Phi\left(\sigma\left(\theta, t_{0}, 0\right), t, t_{0}\right) x\right\| \leq N\left(\theta, t_{0}\right) e^{-\nu(\theta)\left(t-t_{0}\right)}\|x\|,
$$

for all $t \geq t_{0}$ and $\theta \in \Theta$.

Now let $x \in X \backslash\{0\}$ such that there exists $t^{\prime}>t_{0}$ and

$$
\Phi\left(\sigma\left(\theta, t_{0}, 0\right), t^{\prime}, t_{0}\right) x=0
$$

It follows that

$$
\Phi\left(\sigma\left(\theta, t_{0}, 0\right), t^{\prime}, 0\right) \Phi\left(\sigma\left(\theta, t_{0}, 0\right), t^{\prime}, t_{0}\right) x=0, \text { for all } t>t^{\prime} .
$$

We denote

$$
\mu=\inf \left\{t>t_{0}: \Phi\left(\sigma\left(\theta, t_{0}, 0\right), t, t_{0}\right) x=0\right\} .
$$

Therefore $\Phi\left(\sigma\left(\theta, t_{0}, 0\right), \mu, t_{0}\right) x=0$ and

$$
\Phi\left(\sigma\left(\theta, t_{0}, 0\right), t, t_{0}\right) x \neq 0,
$$

for all $t \in\left[t_{0}, \mu\right)$. This shows that

$$
\left\|\Phi\left(\sigma\left(\theta, t_{0}, 0\right), t, t_{0}\right) x\right\| \leq N\left(\theta, t_{0}\right) e^{-\nu(\theta)\left(t-t_{0}\right)}\|x\|,
$$

for all $t \geq t_{0}, \theta \in \Theta$ and $x \in X$. 
REMARK 4.4. In the above theorem, if $\sup _{\theta \in \Theta} k(\theta)=k<\infty$ and the pair of function spaces $\left(\mathcal{C}_{00}\left(\mathbb{R}_{+}, X\right), \mathcal{C}\left(\mathbb{R}_{+}, X\right)\right)$ is admissible to $\pi=(\Phi, \sigma)$, then there exist $N: \mathbb{R}_{+} \rightarrow \mathbb{R}_{+}^{*}$ and $\nu=\frac{1}{k}>0$ such that

$$
\| \Phi\left(\sigma\left(\theta, t_{0}, 0\right), t, t_{0} x\left\|\leq N\left(t_{0}\right) e^{-\nu\left(t-t_{0}\right)}\right\| x \|,\right.
$$

for all $t \geq t_{0}, \theta \in \Theta$ and $x \in X$.

\section{REFERENCES}

[1] L. Barreira, D. Dragičević and C. Valls, Characterization of strong exponential dichotomies, Bull. Braz. Math. Soc. (N.S.) 46 (2015), 81-103.

[2] L. Barreira, D. Dragičević and C. Valls, Strong and weak $\left(L^{p}, L^{q}\right)$-admissibility, Bull. Sci. Math. 138 (2014), 721-741.

[3] L. Barreira, D. Dragičević and C. Valls, Exponential dichotomies with respect to a sequence of norms and admissibility, Internat. J. Math. 25 (2014), 1450024, 20 pp.

[4] C. Chicone and Y. Latushkin, Evolution semigroups in dynamical systems and differential equations, AMS, Providence, 1999.

[5] S.-N. Chow and H. Leiva, Existence and roughness of the exponential dichotomy for skew-product semiflow in Banach spaces, J. Differential Equations 120 (1995), 429477.

[6] S.-N. Chow and H. Leiva, Two definitions of exponential dichotomy for skew-product semiflow in Banach spaces, Proc. Amer. Math. Soc. 124 (1996), 1071-1081.

[7] W. A. Coppel, Dichotomies in stability theory, Springer-Verlag, Berlin-New York, 1978.

[8] J. L. Daleckij and M. G. Krĕ̌n, Stability of differential equations in Banach space, Amer. Math. Soc., Providence, Rhode Island, 1974.

[9] P. Hartman, Ordinary differential equations, Wiley, New-York, London, Sydney, 1964.

[10] Yu. D. Latushkin and A. M. Stëpin, Linear skew-product flows and semigroups of weighted composition operators, in Lyapunov exponents, Springer-Verlag, New-York, 1991.

[11] Y. Latushkin and R. Schnaubelt, Evolution semigroups, translation algebra and exponential dichotomy of cocycles, J. Differential Equations 159 (1999), 321-369.

[12] J. L. Massera and J. J. Schäffer, Linear differential equations and functional analysis. I, Ann. Math. (2) 67 (1958), 517-573.

[13] J. L. Massera and J. J. Schäffer, Linear differential equations and function spaces, Academic Press, New-York, 1966.

[14] M. Megan and C. Stoica, Exponential instability of skew-evolution semiflows in Banach spaces, Stud. Univ. Babeş-Bolyai Math. 53 (2008), 17-24.

[15] O. Perron, Die Stabilitätsfrage bei Differentialgleichungen, Math. Z. 32 (1930), 703728.

[16] C. Preda, P. Preda and A. Petre, On the asymptotic behavior of an exponentially bounded, strongly continuous cocycle over a semiflow, Commun. Pure Appl. Anal. 8 (2009), 1637-1645.

[17] P. Preda, A. Pogan and C. Preda, Schäffer spaces and uniform exponential stability of linear skew-product semiflows, J. Diff. Eq. 212(2005), 191-207.

[18] M. Reghiş, On nonuniform asymptotic stability, Prikl Math. Meh. 27 (1963), 231243 (Russian) [English transl. J. Appl. Math. Mech. 27(1963), 344-362].

[19] M. Rasmussen, Dichotomy spectra and Morse decompositions of linear nonautonomous differential equations, J. Differential Equations 246 (2009), 2242-2263. 
[20] R. J. Sacker and G. R. Sell, Dichotomies for linear evolutionary equations in Banach spaces, J. Differential Equations 113 (1994), 17-67.

[21] G. R. Sell and Y. You, Dynamics of evolutionary equations, Springer Verlag, NewYork, 2002.

C. Preda

Department of Economics and Business Modeling Faculty of Economics and Business Administration

West University of Timişoara

300115 Timişoara

Romania

E-mail: ciprian.preda@e-uvt.ro

S. Rămneanţu

Department of Mathematics

Faculty of Mathematics and Computer Science

West University of Timişoara

300115 Timişoara

Romania

E-mail: ramneantusebastian@yahoo.com

R. Mureşan

Department of Computer Science

Faculty of Mathematics and Computer Science

West University of Timişoara

300115 Timişoara

Romania

E-mail: raluca.muresan@e-uvt.ro

Received: 5.6.2015.

Revised: 28.8.2015. 